\title{
Soil Support Characterization in Slab-on-Grade Constructions with Fiber-Optic Distributed Strain Sensing
}

\author{
Levenberg, Eyal; Klar, Assaf-Avraham; Skar, Asmus
}

Published in:

Geo-Congress 2020: Foundations, Soil Improvement, and Erosion

Link to article, DOI:

$10.1061 / 9780784482780.036$

Publication date:

2020

Document Version

Early version, also known as pre-print

Link back to DTU Orbit

Citation (APA):

Levenberg, E., Klar, A-A., \& Skar, A. (2020). Soil Support Characterization in Slab-on-Grade Constructions with Fiber-Optic Distributed Strain Sensing. In J. P. Hambleton, R. Makhnenko, \& A. S. Budge (Eds.), Geo-Congress 2020: Foundations, Soil Improvement, and Erosion (pp. 370 - 378). American Society of Civil Engineers. https://doi.org/10.1061/9780784482780.036

\section{General rights}

Copyright and moral rights for the publications made accessible in the public portal are retained by the authors and/or other copyright owners and it is a condition of accessing publications that users recognise and abide by the legal requirements associated with these rights.

- Users may download and print one copy of any publication from the public portal for the purpose of private study or research.

- You may not further distribute the material or use it for any profit-making activity or commercial gain

- You may freely distribute the URL identifying the publication in the public portal 


\title{
Soil Support Characterization in Slab-On-Grade Constructions with Fiber- Optic Distributed Strain Sensing
}

\author{
Eyal Levenberg ${ }^{1}$, Assaf A. Klar ${ }^{2}$, and Asmus Skar ${ }^{3}$ \\ ${ }^{1}$ The Technical University of Denmark (DTU), Department of Civil Engineering, Brovej 118, \\ Kongens Lyngby 2800, Denmark; e-mail: eylev@byg.dtu.dk \\ ${ }^{2}$ The Technical University of Denmark (DTU), Department of Civil Engineering, Brovej 118, \\ Kongens Lyngby 2800, Denmark; e-mail: askla@byg.dtu.dk \\ ${ }^{3}$ The Technical University of Denmark (DTU), Department of Civil Engineering, Brovej 118, \\ Kongens Lyngby 2800, Denmark; e-mail: asska@ byg.dtu.dk
}

\begin{abstract}
This study offered a novel method for characterizing the soil support in slab-on-grade constructions such as rigid pavements and raft foundations. The method applies to slabs that are instrumented with fiber-optic cables for distributed strain sensing; it is based on analyzing the spatial profiles of slab bending strains generated by randomly applied surface loads. The concept was demonstrated for a synthetic case involving an infinite plate resting on a Pasternak support model. The method was shown to deliver non-destructive, non-disruptive, and load-independent quantitative information on the prevailing soil support. Therefore, it is deemed well suited for longterm health monitoring applications of slab-on-grade constructions. Such monitoring can help facility owners evaluate and intercept external events that undermine the integrity of the structure. In the long-term, continued monitoring of soil support can help the engineering community improve analysis approaches and design decisions concerning soil-structure interaction.
\end{abstract}

\section{INTRODUCTION}

Common civil engineering structures such as rigid pavements and raft foundations are essentially slab-on-grade constructions, comprised of precast or cast-in-place Portland cement concrete slabs supported on a soil substrate. In the vast majority of cases, the mechanical behavior of the slabs conforms to the classical Germain-Kirchhoff plate theory, i.e., uniform thickness, linear elastic isotropic properties, small deflections relative to the thickness, and small deflection-slopes relative to unity (Timoshenko and Woinowsky-Krieger 1959). The support offered by the soil is usually represented by a Winkler spring-bed model (Hemsley, 1999), characterized by a single parameter called the coefficient of subgrade reaction (units of force/length ${ }^{3}$ ). Incidentally, such representation is mathematically equivalent to the support offered at the surface of a Gibson soil model comprising of a non-homogenous linear elastic half-space characterized by a Young's modulus that linearly increases with depth (starting with zero at the surface) and a Poisson's ratio of 0.5 (Gibson 1967). 
Yet another classically accepted soil support model, though less utilized in standard practice, is that of Pasternak (Pasternak 1954), wherein a non-compressible layer is added on top of the Winkler model for introducing shear interaction between the individual springs. Mathematically, such a model is equivalent to a so-called Vlasov support model, which comprises of a linear elastic (isotropic) layer of finite thickness resting on an infinitely stiff half-space (Vlasov and Leontiev 1966, Jones and Xenophonto 1977). Vlasov's model relates between the spring properties and the superimposed layer through a shape function and energy principle of a constrained elastic domain.

In slab-on-grade constructions, the plate properties are essentially engineered and can therefore be chosen a priori. Moreover, these properties are physically meaningful and hence directly measurable from material element tests. For soil support models, however, the situation is much different. In effect, it is rather difficult to identify the most suitable model in a given situation, and even if identified, it is hard to assign representative values to the governing parameters. One reason for these difficulties is the highly idealized representation, which introduces model parameters that do not directly correspond to any basic soil properties. This very aspect was the impetus for Tezaghi's classical paper on the coefficient of subgrade reaction (Terzaghi 1955); it also spurred a myriad of subsequent studies dealing with appropriate selection of soil support parameters (Vesić 1961, Chou 1983, Ioannides 1985, Fwa et al. 1996, Daloglu and Vallabhan 2000). Another reason is the sensitivity of the support characteristics to environmental conditions. Unlike slab properties that remain relatively stable throughout the life of the structure (or evolve in a manner that is fairly well understood), the soil responses continually evolve under service due to changes in density, temperature, moisture, and time (age). Consequently, for a given slab-on-grade construction at a given time, it is not straightforward to identify the governing support model type, and it is not simple to quantify the support parameters.

The traditional approach for soil support characterization is based on full-scale loading experiments (Setiadji and Fwa 2009, ASTM D1196). These entail application of a load with known dimensions and intensity, and measurement of the resulting mechanical responses at several predetermined locations. By very nature, such procedure is both expensive and service-disruptive, essentially applicable only at sparse time intervals. As a means of addressing these limitations, it is the objective of this work to offer a new method for characterizing the support conditions in slab-on-grade constructions. The purpose of the development is twofold: (i) identify the support model type that best applies in a given situation, i.e., Winkler/Gibson vs. Pasternak/Vlasov, and (ii) characterize the associated support properties, i.e., quantify the coefficient of subgrade reaction and the intensity of shear interaction between the Winkler springs.

Fundamental to the proposed approach is the assumption that slabs, having known properties, are instrumented with Fiber-Optic (FO) cables for distributed strain sensing. Such technology is being introduced into civil engineering infrastructure in recent years as a means of transforming conventional constructions into intelligent or self-aware constructions (Klar et al. 2016a, Klar et al. 2016b, Barrias et al. 2016). Accordingly, the method is based on analyzing the spatial strain profiles (due to plate bending) measured by embedded FO cables. The strains being 
targeted are those that are generated by surface loadings to which the structure is exposed as part of its normal service conditions. A unique feature of the proposed approach in this connection is that loading dimensions and loading intensities are not required inputs. Thus, the overall aim herein is to offer a soil support characterization approach for slab-on-grade constructions that is nondestructive, non-disruptive, and load-independent.

The paper commences with restating the basic slab-on-grade formulation for an infinite plate on a Pasternak support that is loaded uniformly over a circular area. Then, the new soil support characterization method is formulated based on a theoretical analysis of plate bending strain-profiles expected under different support conditions. The paper concludes with a short discussion of the approach, followed by some suggestions for further work.

\section{SLAB-ON-GRADE FORMULATION}

Consider a Germain-Kirchhoff plate of infinite size characterized by thickness $h$, Young's modulus $E$, Poisson's ratio $v$, and therefore flexural rigidity $D=E h^{3}\left(1-v^{2}\right)^{-1} / 12$. The plate is loaded at the surface by a uniform vertical stress with intensity $q$ distributed over a circular area with radius $a$. Given that this is an axisymmetric situation, the vertical displacement field $u_{z}$ only depends on the radial coordinate $r$ with origin located under the load centroid, i.e., $u_{z}=u_{z}(r)$.

In the case of a Pasternak support type, the vertical displacement field is given by (Van Cauwelaert et al. 2002, Khazanovich and Levenberg 2018)

$$
u_{z}=\frac{q a}{l k} \int_{0}^{\infty} \frac{J_{0}(m r / l) J_{1}(m a / l)}{m^{4}+2 b m^{2}+1} d m
$$

where $l=\sqrt[4]{D / k}$ is the characteristic length or the so-called radius of relative stiffness (Westergaard 1926), $k$ is the coefficient of subgrade reaction, $J_{n}$ denotes a Bessel function of the first kind of order $n, m$ is a unitless integration parameter (or wave number), and $b=G\left(2 k l^{2}\right)^{-1}$ is a unitless Pasternak parameter (positive) wherein $G$ (units of force/length) represents the intensity of shear interaction between the Winkler springs. The special case of $b=0$, which is equivalent to $G=0$, provides the solution for a plate on a Winkler support. As pointed out in Kerr (1965), in realistic slab-on-grade constructions the system response to load is dominated by vertical stiffness and not shear interaction effects; this essentially means that $b \leq 1$.

The plate's radial and tangential moments per unit length, $M_{r}$ and $M_{\theta}$ (respectively), are obtained from the usual expressions

$$
M_{r}=-D\left(\frac{d^{2} u_{z}}{d r^{2}}+\frac{v}{r} \frac{d u_{z}}{d r}\right)
$$




$$
M_{\theta}=-D\left(\frac{1}{r} \frac{d u_{z}}{d r}+v \frac{d^{2} u_{z}}{d r^{2}}\right)
$$

Upon insertion of Equation (1) into Equations (2) and (3), expressions for plate's bending moments are obtained

$$
\begin{aligned}
& M_{r}=\frac{q a D}{l^{2} k r} \int_{0}^{\infty} \frac{m J_{1}(m a / l)\left((m r / l) J_{0}(m r / l)-(1-v) J_{1}(m r / l)\right)}{m^{4}+2 b m^{2}+1} d m \\
& M_{\theta}=\frac{q a D}{l^{2} k r} \int_{0}^{\infty} \frac{m J_{1}(m a / l)\left((v m r / l) J_{0}(m r / l)+(1-v) J_{1}(m r / l)\right)}{m^{4}+2 b m^{2}+1} d m
\end{aligned}
$$

The corresponding radial and tangential bending stresses are $\sigma_{r}=12 z M_{r} / h^{3}$ and $\sigma_{\theta}=12 z M_{\theta} / h^{3}$ (respectively) where $z$ is measured vertically downward from the plate's mid-surface (or neutral plane) towards the support. Thus, the extremal bending stresses are obtained at the surface where $z=-h / 2$ and at the bottom of the plate where $z=h / 2$. The chosen notation is such that a positive moment is associated with tensile bending stress (or strain) at the bottom of the plate below the loaded area, i.e., where $r=0$ and $z=h / 2$. Finally, the radial strain $\varepsilon_{r}$ at a given $z$ is obtained from

$$
\varepsilon_{r}=\frac{\sigma_{r}-v \sigma_{\theta}}{E}
$$

\section{PROPOSED CHARACTERIZATION METHOD}

To best explain the soil support characterization idea advocated herein, consider an infinite plate instrumented with FO cables for distributed strain sensing; the cables are envisioned to be deployed in a dense mesh and positioned close to the bottom of the slab. It is also assumed that the fibers are flexible to such an extent that they do not reinforce the plate and alter its flexural rigidity. Whenever a single random load, having unknown radius and intensity, is applied at the plate's surface, the sensing array should be capable of measuring the radial strain profile induced by the load. Figure 1 provides a generic illustration of the expected measurement, applicable to both Winkler and Pasternak models. The ordinate in this figure depicts $\varepsilon_{r}$ based on Equation (6) with $z=h / 2$, while the abscissa depicts radial offset relative to the location of load application, normalized by the characteristic length $l$. 


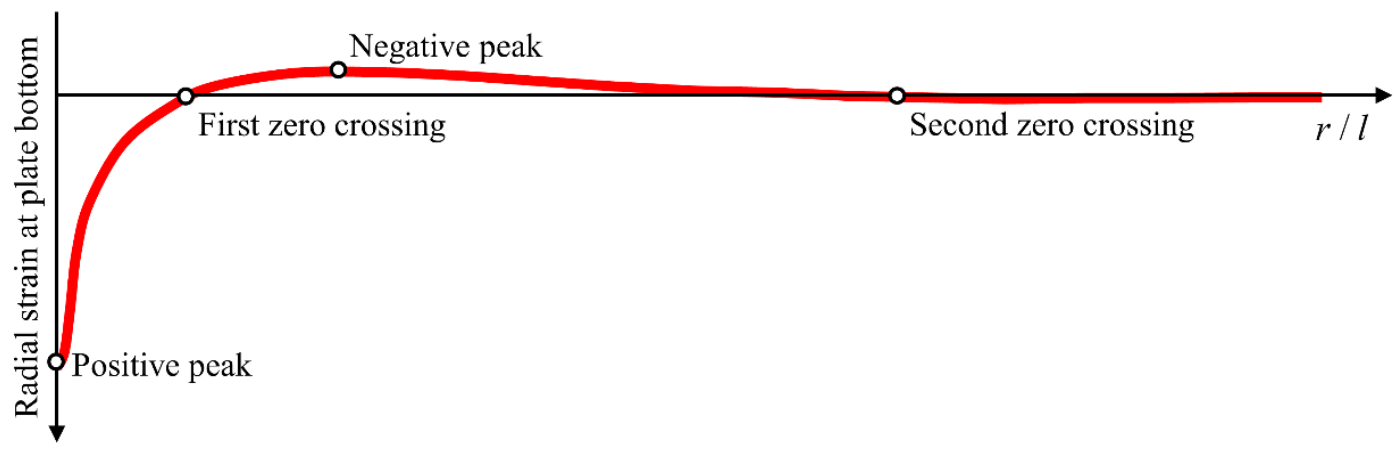

Figure 1. Generic illustration of the radial strain profile along the bottom of a loaded plate.

Figure 1 shows how the radial strain decreases from a positive (tensile) peak level, attained directly below the load centroid, to zero at some radial distance (first zero crossing). With larger radial offsets the strain further decreases to negative values. At a certain radial offset a negative peak is attained, beyond which the strain increases again and becomes positive. Thus, a second zero crossing occurs. In theory, more zero strain crossings are anticipated for larger radial offsets. However, the overall strain level becomes very small - too small to enable the reliable identification of subsequent zero crossings.

The location of the first and second zero crossings, as well as the location of the negative peak, are considered Points Of Interest (POIs). This is because their relative locations are closely related to the governing support model type and to the numerical values of the model parameters. To demonstrate this statement, the abovementioned plate theory was interrogated to identify the radial offsets for the POIs in Figure 1. This was done for two very different cases, spanning two extreme yet realistic situations. The first case considered a thick concrete plate with $h=500 \mathrm{~mm}$ resting on a 'soft' spring-bed support characterized by $k=0.02 \mathrm{MPa} / \mathrm{mm}$. The second case considered a thin concrete plate with $h=100 \mathrm{~mm}$ resting on a 'hard' spring-bed support characterized by $k=0.20 \mathrm{MPa} / \mathrm{mm}$. Taking accepted concrete properties $E=30 \mathrm{GPa}$ and $v=0.15$, Case I represents a relatively large characteristic length of $l=2000 \mathrm{~mm}$, and Case II represents a relatively small characteristic length of $l=336 \mathrm{~mm}$.

Presented in Table 1 are calculated radial offsets normalized with respect to the characteristic length for the POIs in the two cases. The first row of calculated results is associated with $b=0$, and therefore refers to a Winkler model. The successive rows refer to a Pasternak support model with increasing shear parameter values up to $b=1$ or equivalently up to $G=2 k l^{2}$. Calculations were performed with $a=150 \mathrm{~mm}$; however this choice is deemed inconsequential because, as was observed in a separate analysis, results are essentially insensitive to the exact radius of loading as long as it is of the same order as the plate thickness or smaller (down to a point load). The results in Table 1 were also found to be essentially insensitive to the value of $v$. Moreover, it is important to note that the loading intensity $q$ has no effect on the shown results.

Obtaining Table 1 values required numerical evaluation of the integrals in Equations (4) and (5). To achieve this, the semi-infinite integration range (over $m$ ) was truncated to include the 
first 100,000 integrand roots. Then, a 30-point Gauss quadrature method was applied to each of the intervals between the successive roots. With this procedure, radial strains were calculated at $0.1 l$ intervals from $r=0$ and up to $r=25 l$. Finally, a cubic spline interpolation scheme was utilized to generate a simpler analytic representation of the entire strain profile shape, from which the normalized radial offsets of the POIs were ultimately obtained.

Figure 2 provides a graphical representation of Table 1 values with $b$ as the abscissa. It can be seen that the results for the two cases essentially overlap, i.e., that the normalized radial offsets of the POIs are relatively insensitive to the value of $l$. The first zero crossing location is influenced by $b$, dropping from about $0.86 l$ at $b=0$ to about $0.67 l$ at $b=1$. The negative peak location also drops with increasing $b$, from about $1.86 l$ at $b=0$ to about $1.40 l$ when $b=1$. A pronounced dependence on $b$ is exhibited by the location of the second zero crossing, increasing from about $5.98 l$ at $b=0$ towards infinity as $b$ approaches unity.

Figure 3 presents the location ratio of the first to second zero crossings as a function of Pasternak's $b$ parameter. Two curves are shown, each associated with a different case in Table 1 . While the two curves do not collapse onto one unique line, the gap between them is considered small, establishing an almost unique relationship between the abovementioned ratio and Pasternak's parameter.

Table 1. Normalized radial offsets of the POIs (refer to Figure 1) for two distinct cases.

\begin{tabular}{|c|c|c|c|c|c|c|}
\hline & \multicolumn{2}{|c|}{ Case I, $l=2000 \mathrm{~mm}$} & \multicolumn{3}{c|}{ Case II, $l=336 \mathrm{~mm}$} \\
\hline $\begin{array}{l}\text { Value of } b \text { in } \\
\text { Equation (1) }\end{array}$ & $\begin{array}{c}\text { First } \\
\text { zero } \\
\text { crossing }\end{array}$ & $\begin{array}{c}\text { Negative } \\
\text { peak }\end{array}$ & $\begin{array}{c}\text { Second } \\
\text { zero } \\
\text { crossing }\end{array}$ & $\begin{array}{c}\text { First } \\
\text { zero } \\
\text { crossing }\end{array}$ & $\begin{array}{c}\text { Negative } \\
\text { peak }\end{array}$ & $\begin{array}{c}\text { Second } \\
\text { zero } \\
\text { crossing }\end{array}$ \\
\hline $\mathbf{0 . 0 0}$ & 0.812 & 1.822 & 5.962 & 0.903 & 1.893 & 5.997 \\
\hline $\mathbf{0 . 2 0}$ & 0.754 & 1.696 & 6.456 & 0.857 & 1.779 & 6.494 \\
\hline $\mathbf{0 . 4 0}$ & 0.706 & 1.587 & 7.202 & 0.819 & 1.681 & 7.244 \\
\hline $\mathbf{0 . 6 0}$ & 0.665 & 1.492 & 8.478 & 0.787 & 1.598 & 8.521 \\
\hline $\mathbf{0 . 7 0}$ & 0.647 & 1.449 & 9.561 & 0.773 & 1.560 & 9.606 \\
\hline $\mathbf{0 . 8 0}$ & 0.630 & 1.409 & 11.385 & 0.761 & 1.526 & 11.431 \\
\hline $\mathbf{0 . 8 5}$ & 0.622 & 1.390 & 12.924 & 0.755 & 1.509 & 12.970 \\
\hline $\mathbf{0 . 9 0}$ & 0.615 & 1.371 & 15.507 & 0.749 & 1.493 & 15.554 \\
\hline $\mathbf{0 . 9 5}$ & 0.607 & 1.353 & 21.361 & 0.743 & 1.477 & 21.414 \\
\hline $\mathbf{1 . 0 0}$ & 0.600 & 1.336 & $\infty$ & 0.738 & 1.463 & $\infty$ \\
\hline
\end{tabular}




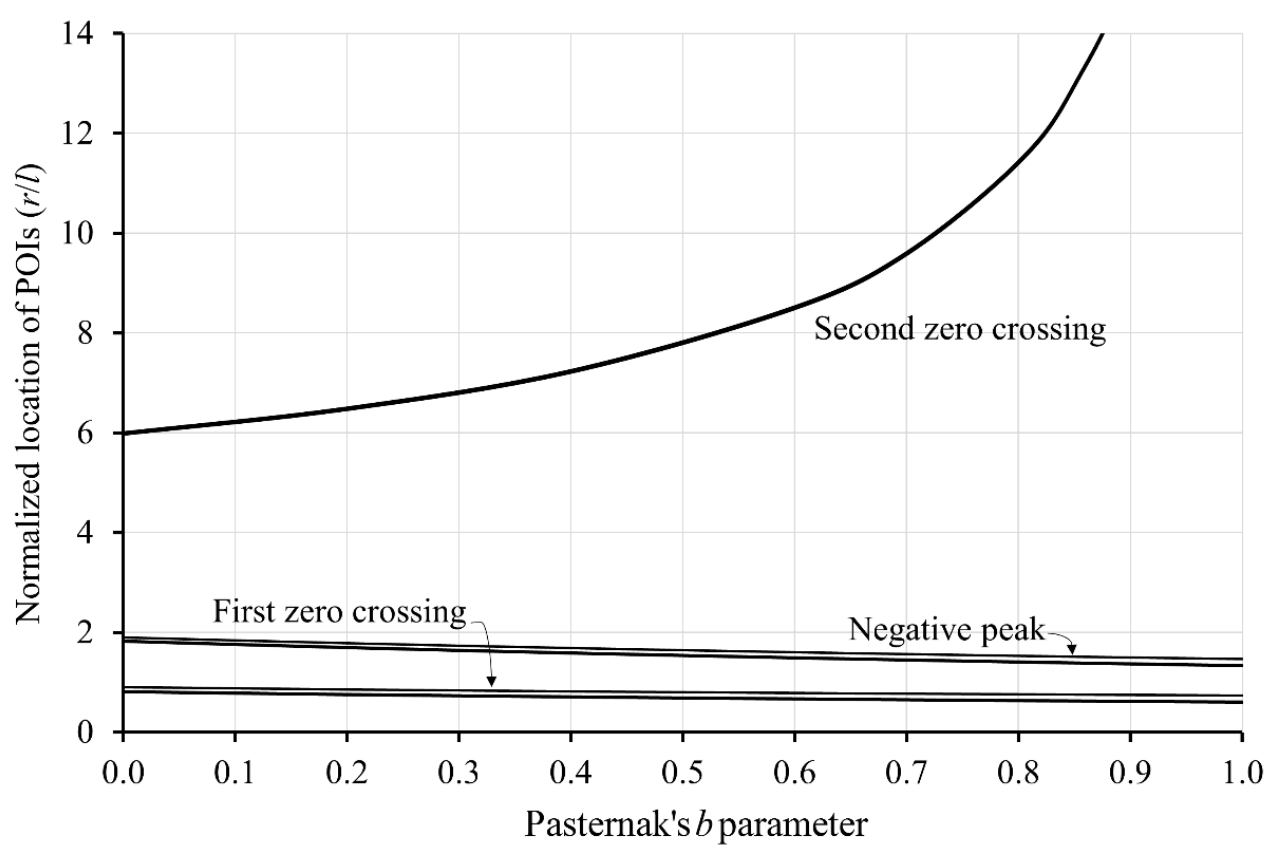

Figure 2. Influence of Pasternak's $b$ parameter on the normalized locations of the POIs defined in Figure 1 for two Cases considered in Table 1.

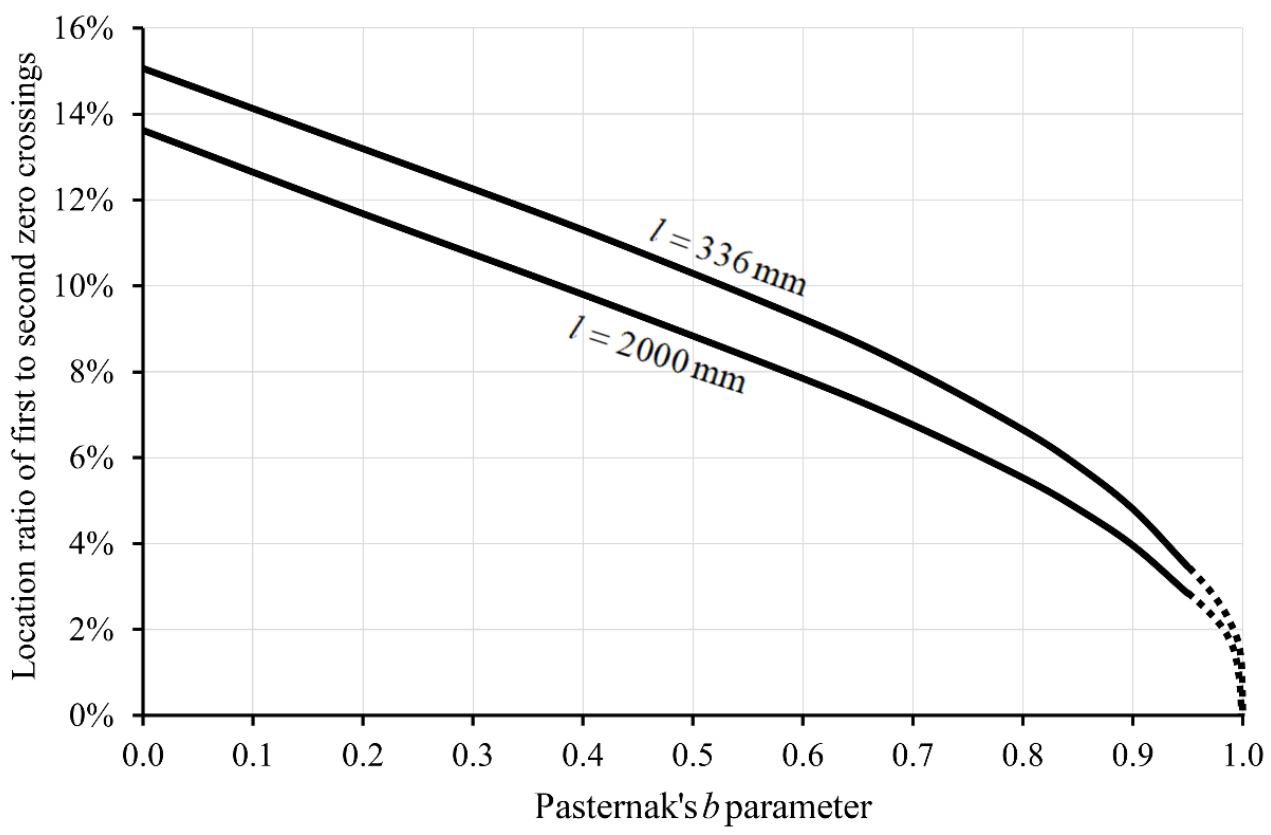

Figure 3. Influence of Pasternak's $b$ parameter on the location ratio of first to second zero crossings defined in Figure 1 for the two cases considered in Table 1.

Ultimately, Figures 2 and 3 offer new means for performing soil support characterization for slab-on-grade constructions. First, this means identifying whether a Winkler model or a Pasternak model should be applied to represent the support the substrate provides to the slab at the 
point in time when the evaluation is performed. Second, this means estimating the numerical values of the governing model parameters - the coefficient of subgrade reaction, and if relevant the intensity of shear interaction between the Winkler springs (i.e., Pasternak's parameter). The starting point for the method is identifying the locations of the POIs (see Figure 1) from FO strain sensing. This step is non-destructive, non-disruptive, load-independent, and can be done repeatedly under usual service conditions - whenever desired. Next, Figure 3 is entered with the location ratio of the first to second zero crossings to provide a range of estimated values for Pasternak's $b$ parameter. The average of this range is then utilized in Figure 2 to resolve the value of the characteristic length $l$. Once $l$ is identified, a second iteration is to be carried out to refine the estimation of $b$ and subsequently the estimation of $l$. Finally, given that the plate properties are known, it becomes possible to calculate the coefficient of subgrade reaction $k=D l^{-4}$, and to calculate the intensity of shear interaction between the Winkler springs $G=2 b k l^{2}$. When the above-described procedure is performed at different spatial locations across the slab-on-grade construction, and at different times during the service life of the system, it becomes possible to track the influence of external events (e.g., nearby tunneling or unexpected weather conditions) on the support behavior and eventually on the structural response. It also become possible to better understand the reasons for any observed spatial and temporal variability in performance.

To exemplify the method, consider a concrete plate with $h=220 \mathrm{~mm}, E=35 \mathrm{GPa}$, and $v=0.20$. The plate is resting on an unknown soil support type and loaded by a single load with unknown radius and unknown contact stress intensity. An embedded FO sensing array measures the following offset values for the POIs: radial location of first zero crossing $554 \mathrm{~mm}$, radial location of negative peak $1238 \mathrm{~mm}$, and radial location of second zero crossing $5142 \mathrm{~mm}$. Based on the measurements, the ratio of first zero crossing to second zero crossing is seen to be $10.8 \%$. Based on Figure 3, this ratio corresponds to a Pasternak parameter between $b=0.297$ (for $l=2000 \mathrm{~mm}$ ) and $b=0.451$ (for $l=336 \mathrm{~mm}$ ). Next, Figure 2 is entered with an average value of $b=0.374$ to provide the following three different estimates, based on the three different curves: (i) the first zero crossing occurs at a radial offset equal to $0.768 l$, (ii) the negative peak occurs at a radial offset equal to $1.647 l$, and (iii) the second zero crossing occurs at a radial offset equal to 7.126l. Consequently, the characteristic length is estimated, via averaging of the three different possible values, to be $732 \mathrm{~mm}$. At this point, a second iteration is performed; i.e., Figure 3 is entered again with $10.8 \%$, and a value of $b=0.334$ is obtained via interpolation between the two curves assuming that $l=732 \mathrm{~mm}$. Next, Figure 2 is reentered, this time with $b=0.334$, and the above-described calculations are repeated. The final result is $l=731 \mathrm{~mm}$ (practically identical to the first iteration result) which leads to a modulus of subgrade reaction of $k=0.11 \mathrm{~N} / \mathrm{mm}^{3}$ and (because $b=0.334$ ) to a shear interaction intensity between the individual springs of $G=40.4 \mathrm{kN} / \mathrm{mm}$. The synthetic example was prepared with $k=0.10 \mathrm{~N} / \mathrm{mm}^{3}$ (i.e., $l=754 \mathrm{~mm}$ ), $G=35 \mathrm{kN} / \mathrm{mm}$ (i.e., $b=0.308$ ), and $a=100 \mathrm{~mm}$. Thus, after two iterations, $b$ was estimated within $7.6 \%$ accuracy and $l$ within $3.1 \%$ accuracy. 


\section{CONCLUSION}

This study offered a new and novel method for characterizing the resilient soil support in slab-ongrade constructions. The method allows for non-destructive, non-disruptive, and load-independent identification of the governing support model type (Winkler vs. Pasternak) and quantification of the underlying parameter values, i.e., Winkler's coefficient of subgrade reaction and Pasternak's parameter - refer to Equation (1). The idea applies to slabs that are instrumented with FO cables for distributed strain sensing; it is based on the availability of continuous structural response information, which is not accessible from the measurements of 'point' sensors. The characterization is based on analyzing the spatial shape features of slab bending strains due to a randomly applied surface load (i.e., peak locations and zero crossings), and not on the strain magnitudes. In principle, every new loading event can trigger a new calculation. Doing so will not only reduces random measurement errors, it will also provide the evolution of the prevailing soil support behavior in a given situation. Such capabilities are useful for facility owners, to help evaluate and intercept external events that can potentially undermine the integrity of the structure. In the long-term, they are also useful to the engineering community - for improving analysis approaches and design decisions concerning soil-structure interaction.

In order for the method to gain practical acceptance, more development work is needed, e.g., (i) assessing the realistic capability of distributed strain-sensing technologies to identify the POIs in Figure 1; (ii) expanding the theory to relieve the infinite slab assumption by including free plate edges and the effects of shear transfer across joints; (iii) addressing realistic situations where the measured strains are due to multiple random loads the are simultaneously applied at the surface; and (iv) experimentally validating the idea in a small-scale setup, followed by a prototype-scale demonstration. These suggestions will serve as topics for future papers.

\section{REFERENCES}

ASTM D1196 (2016). Standard Test Method for Nonrepetitive Static Plate Load Tests of Soils and Flexible Pavement Components, for Use in Evaluation and Design of Airport and Highway Pavements. ASTM International, West Conshohocken, PA, 2016, www.astm.org.

Barrias, A., Casas, J., and Villalba, S. (2016). "A Review of Distributed Optical Fiber Sensors for Civil Engineering Applications.” Sensors, 16(748), 1-35.

Chou, Y. T. (1983). "Subgrade Contact Stresses under Rigid Pavements." Journal of Transportation Engineering, 109(3), 363-379.

Daloglu, A. T. and C. V. G. Vallabhan, (2000). "Values of k for Slab on Winkler Foundation." Journal of Geotechnical and Geoenvironmental Engineering, 126, 463-471.

Fwa, T. F., Shi, X. P., and Tan, S. A. (1996). "Use of Pasternak Foundation Model in Concrete Pavement Analysis.” Journal of Transportation Engineering, 122(4), 323-328.

Gibson, R. E. (1967). "Some Results Concerning Displacements and Stresses in a Nonhomogenous Elastic Half-space." Geotechnique, 17, 58-67. 
Hemsley J. A. (1999). Elastic Analysis of Raft Foundations. Thomas Telford, London.

Ioannides, A. M. (1985). "Subgrade Contact Stresses under Rigid Pavements: Discussion.” Journal of Transportation Engineering, 111(3), 321-326.

Jones, R. and Xenophontos, J. (1977). “The Vlasov Foundation Model.” International Journal of Mechanical Sciences, 19, 317-323.

Kerr, A. D. (1965). "Continuity in Foundation Models and Related Problems." Research Report 109, DA Task IV025001A13001, Cold Regions Research and Engineering Laboratory (CRREL), Hanover, New Hampshire, USA.

Khazanovich, L. and Levenberg, E. (2018). "Analytical Solution for a Viscoelastic Plate on a Pasternak Foundation." Road Materials and Pavement Design, DOI:10.1080/14680629.2018.1530693.

Klar, A., Levenberg, E., Tur. M., and Zadok, A. (2016a). "Sensing for Smart Infrastructure: Prospective Engineering Applications." Proceedings of the International Conference on Smart Infrastructure and Construction, Mair, R.J., Soga, K., Jin, Y., Parlikad, A.K. and Schooling, J.M. (eds.), Institution of Civil Engineers (ICE) Publishing, London, United Kingdom, 289-295.

Klar, A., Uchida, S., and Levenberg, E. (2016b). "In Situ Stiffness Profiling using High Resolution Fiber Optic Distributed Sensing." Journal of Geotechnical and Geoenvironmental Engineering, 142(8), 04016032(1-9).

Pasternak, P. L. (1954). "On a New Method of Analysis of an Elastic Foundation by Means of Two Constants." Gosudarstvennoe Izdatelstvo Literaturi po Stroitelstvui Arkhitekture, Moscow - in Russian.

Setiadji, B. H. and Fwa, T. F. (2009). "Examining k-E Relationship of Pavement Subgrade Based on Load-Deflection Consideration.” Journal of Transportation Engineering, 135(3), 140148.

Terzaghi, K. (1955). "Evaluation of Coefficient of Subgrade Reaction.” Geotechnique, 5(4), 297326.

Timoshenko, S. P., and Woinowsky-Krieger, S. (1959). Theory of plates and shells; $2^{\text {nd }}$ edition. McGraw-Hill, New York.

Van Cauwelaert, F., Stet, M., and Jasienski, A. (2002). "The General Solution for a Slab Subjected to Centre and Edge Loads and Resting on a Kerr Foundation.” International Journal of Pavement Engineering, 3(1), 1-18.

Vesić B. S. (1961). "Beams on Elastic Subgrade and Winkler's Hypothesis." Proceedings of the 5th International Conference on Soil Mechanics and Foundation Engineering, Vol. 1, 845850.

Vlasov, V. Z. and Leontiev, U. N. (1966). Beams, Plates, and Shells on Elastic Foundation. Translated from Russian by the Israel Program for Scientific Translations, Jerusalem.

Westergaard, H. M. (1926). "Stresses in Concrete Pavements Computed by Theoretical Analysis." Public Roads, 7(2), 25-35. 